\title{
ICA based Image denoising for Single-Sensor Digital Cameras
}

\author{
Shawetangi kala ${ }^{1}$
}

Raj kumar sahu ${ }^{2}$

\begin{abstract}
MOST existing digital color cameras use a single sensor with a color filter array (CFA) to capture visual scenes in color. Since each sensor cell can record only one color value, the other two missing color components at each position need to be interpolated. The color interpolation process is usually called color demosaicking (CDM). The quality of demosaicked images is degraded due to the sensor noise introduced during the image acquisition process. The conventional solution of combating CFA sensor noise is demosaicking first, followed by a separate denoising processing. This strategy will generate many noise-caused color artifacts in the demosaicking process, which are hard to remove in the denoising process. Many advanced denoising algorithms, which are designed for monochromatic (or full color) images, are not directly applicable to CFA images due to the underlying mosaic structure of CFAs. A well designed "denoising first and demosaicking later" scheme can have advantages such as less noise-caused color artifacts and cost-effective implementation. In this paper, a single channel ICA based image denoising algorithm is proposed by constructing a noise image to as another observation signal for single channel noise reduction based on independent component analysis, thereby noise and original image can be separated through independent component analysis. Simulation result shows that much better denoising effect and signal-noise ratio can be obtained by using this algorithm.
\end{abstract}

Index Terms: Color filter array (CFA), demosaicking, transform domain, independent component analysis (ICA).

\section{INTRODUCTION}

Digital images play an important role both in daily life applications such as satellite television, magnetic resonance imaging, computer tomography as well as in areas of research and technology such as geographical information systems and astronomy. Data sets collected by image sensors are generally contaminated by noise. Imperfect instruments, problems with the data acquisition process, and interfering natural phenomena can all degrade the data of interest. Furthermore, noise can be introduced by transmission errors and compression. Thus, denoising is often a necessary and the first step to be taken before the images data is analyzed. It is necessary to apply an efficient denoising technique to compensate for such data corruption. Image denoising still remains a challenge for researchers because noise removal introduces artifacts and causes blurring of the image. Most existing digital color cameras use a single sensor with a color filter array (CFA) [1] to capture visual scenes in color. Since each sensor cell can record only one color value, the other two missing color components at each position need to be interpolated from the available CFA sensor readings to reconstruct the full-color image. The color interpolation process is usually called color demosaicking (CDM).Many CDM algorithms [2]-[8] proposed in the past are based on the unrealistic assumption of noise-free CFA data.The presence of noise in CFA data not only deteriorates the visual quality of captured images, but also often causes serious demosaicking artifacts which can be extremely difficult to remove using a subsequent denoising process. Many advanced denoising algorithms which are designed for monochromatic (or full color) images, are not directly applicable to CFA images due to the underlying mosaic structure of CFAs.All these traditional methods consider the signal and noise as the same status but not consider the independence of signal and noise, so they may tend to affect the denoising effect, while independent component analysis (ICA) renders the images as statistically independent as possible by evaluating higher-order statistics of observation images, so it performs well in noise removal and it preserves edge sharpness.

ICA is a novel statistical technique in signal processing and machine learning that aims at finding linear projections of the data that maximize their mutual independence. Its main applications are blind source separation (BSS) and feature extraction. In recent years, ICA has been attracted a lot of attention in image processing application such as multiple channels images blind separation and images feature extraction [9]-[11]. Traditional ICA has some assumption such as the number of sensors is not less than that of original signals, so it can not solve the problem of the source signal number greater than the observation signal number and can not realize single channel image denoising. To overcome the ICA limitation, a single channel image denoising algorithm is presented which believe the image and noise is independent of each other. By estimate a noise properly to as another observation image in ICA, thus solve the problem of single channel blind signal separation and realize image denoising. Separation results obtained from test and real images demonstrate the feasibility of our approach.

\section{Principle of ICA}

ICA is one method for performing blind signal separation that aims to recover unknown sources from a set of their observations, in which they are mixed in an unknown manner. In the basic ICA model, the observed mixture signals $\mathbf{x}(t)$ can be expressed as

$$
\mathbf{x}(t)=\mathbf{A} \mathbf{s}(t)
$$

Where $\mathbf{A}$ is an unknown mixing matrix, and $\mathbf{s}(t)$ represents the latent source signals which are assumed to be statistically mutually independent. The ICA model describes how the observed mixture signals $\mathbf{x}(t)$ are 
generated by a process that uses the mixing matrix $\mathbf{A}$ to mix the latent source signals $\mathbf{s}(t)$ Another concern is the presence of additive noise, which is usually assumed to follow a normal distribution. Denoting the additive noise vector $\mathbf{v}(t)$, then a more realistic and general ICA model is the denoising case:

$$
\mathbf{x}(t)=\mathbf{A s}(t)+\mathbf{v}(t)
$$

The model of noisy ICA can be depicted by Fig.1. In such model, the independent components $\mathbf{s}(t)$ cannot be directly observed and the mixing coefficients $\mathbf{A}$ and the noise $\mathbf{v}(t)$ are also assumed to be unknown. If noise is negligible, only the random variables $\mathbf{x}(t)$ are observed and both the components $\mathbf{S}(t)$ and the coefficients $\mathbf{A}$ must be estimated using $\mathbf{x}(t)$.Then, the ICA solution is obtained in an unsupervised learning process that find a de-mixing matrix $\mathbf{W}$. The matrix $\mathbf{W}$ is used to transform the observed mixture signals $\mathbf{x}(t)$ to yield the independent signals. That is:

$$
\mathbf{s}^{\wedge}(t)=\mathbf{W} \mathbf{x}(t)
$$

The signals $\mathbf{s}^{\wedge}(t)$ are the estimation of the latent source signals $\mathbf{s}(t)$.If $\mathbf{W}=\mathbf{A}^{-}$then the recovered signals $\mathbf{s}^{\wedge}(t)$ are exactly the original sources $\mathbf{s}(t)$. The components of $\mathbf{s}^{\wedge}(t)$ called independent components, are required to be as mutually independent as possible. The aim of presented algorithm is to realize original image and noise separation, so as to realize a single channel image denoising.

There exist several algorithm performing ICA ,from among the several ICA algorithm, we select the FASTICA [12-13] algorithm to use in this study. This algorithm is based on fixed-point method, comparing the algorithms based on gradient descent method, the fixed-point algorithm has a higher speed convergence property since the Newton method in block mode is applied. It is easy to apply in data analysis since there is no learning rate parameter that must be adjusted. Furthermore, we can extract independent components one by one or simultaneously. This means the condition of the prior knowledge of the original signals number will be more relaxed. In the FASTICA algorithm, the initial step is whitening or sphering. This whitening facilitates the separation of the underlying independent signals. By this linear transformation, the observation $\mathbf{x}(t)$ are made uncorrelated and unit-variance. In typical of ICA methods, the whitening may be accomplished by principal component analysis (PCA) projection. Make

$$
\mathbf{x}^{\wedge}(t)=\mathbf{U} \mathbf{x}(t)
$$

Where $\mathbf{x}^{\wedge}(t)$ is the whitening signals with $E\left[\mathbf{x}^{\wedge}(t) \mathbf{x}^{\wedge}(t) \mathrm{T}\right]$ $=\mathbf{I}, \mathrm{U}$ is whitening matrix which is usually computed after singular- or eigen value decomposition of the covariance matrix of $\mathbf{x}(t)$, the process is given by

$$
U=\Lambda^{-1 / 2} \mathbf{V}^{T}
$$

Where $\boldsymbol{\Lambda}=\operatorname{diag}[\lambda(1), \ldots, \lambda(m)]$ is a diagonal matrix with the eigen values of the data covariance matrix $E[\mathbf{x}(t) \mathbf{x}(t)]$ and $\mathbf{V}$ is a matrix with the corresponding eigenvectors as its columns. Substitute Eq.(1) for Eq.(3), the formula becomes:

$$
\hat{\mathbf{x}}(t)=\mathbf{U A s}(t)
$$

And the matrix $\mathbf{B}=\mathbf{U A}$ is orthogonal. Therefore, the solution is now sought in the form:

$$
\mathbf{s}^{\wedge}(t)=\mathbf{W} \mathbf{x}^{\wedge}(t)
$$

If $\mathbf{W}=\mathbf{B}^{-1}$, then the recovered signals $\mathbf{s}^{\wedge}(t)$ are exactly the original signals $\mathbf{s}(t)$.

In order to calculate $\mathbf{W}$, different cost functions are used in the literature, usually involving a nonlinearity that shapes the probability destiny function of the original signals. However high-order statistics, such as the kurtosis, are widely used as well. The kurtosis shows how the independent the signal is because it is the classical measure of non-Gaussianty.

Thus we obtain the FASTICA algorithm as follows:

(1) Center the data to make its mean zero;

(2) Whiten the data to get $\mathbf{x}^{\wedge}(t)$;

(3) Make i=1;

(4) Choose an initial orthogonal matrix for $\mathrm{W}$ and make $\mathrm{k}=1$;

(5) Make

$w_{i}(k)=E\left[\hat{X}_{\mathrm{u}}\left(\left(w_{i}(k-1)^{T} \hat{X}_{\mathrm{i}}\right)^{\mathrm{a}}\right]-3 w_{\mathrm{i}}(k-1)\right.$

(6) Make

$$
w_{i}(k)=\frac{w_{i}(k)}{\left\|w_{i}(k)\right\|}
$$

(7) If not converged, make $\mathrm{k}=\mathrm{k}+1$ and go back to step(5).

(8) Make $\mathrm{i}=\mathrm{i}+1$.

(9) When $i<$ number of original signals, go back to step (4).

\section{Image denoising algorithm based on ICA .}

Image denoising is generally an inverse problem. which intends to recover the original ideal image from its given bad version, such as one that is corrupted by noise. Recently, there are more and more new denoising techniques explored, such as principal components analysis (PCA) approach, independent component analysis (ICA) and standard sparse coding (SC) and so on. These methods can successfully denoise images by using different skills and strategies. While the significant advantage of the PCA and sparse coding is that they depend on the statistic property of data. However, the PCA technique is usually suitable for the second order accumulation variant, whereas the sparse coding and ICA method can be used for multidimensional mixed data. Moreover, when ICA is applied to natural image data, ICA is equivalent to SC. However, ICA emphasizes independence over sparsity in the output coefficients, while SC requires that the output coefficients must be sparse and as independent as possible. So, in recent years, ICA technique has been used widely in multiple channels images denoising field. Moreover here we realize a single channel image denoising using ICA.

\subsection{Colour filter array and Bayerpattern}

A color filter array (CFA), is a mosaic of tiny color filters placed over the pixel sensors of an image sensor to capture color information. Color filters are needed because 
the typical photo sensors detect light intensity with little or no wavelength specificity, and therefore cannot separate color information. Since sensors are made of semiconductors they obey solid-state physics. The color filters filter the light by wavelength range, such that the separate filtered intensities include information about the color of light. For example, the Bayer filter gives information about the intensity of light in red, green, and blue (RGB) wavelength regions. The raw image data captured by the image sensor is then converted to a fullcolor image (with intensities of all three primary colors represented at each pixel) by a demosaicing algorithm which is tailored for each type of color filter. The spectral transmittance of the CFA elements along with the demosaicing algorithm jointly determine the color rendition. The sensor's pass band quantum efficiency and span of the CFA's spectral responses are typically wider than the visible spectrum, thus all visible colors can be distinguished. The responses of the filters do not generally correspond to the CIE color matching functions, so a color translation is required to convert the tristimulus values into a common space .

A Bayer filter mosaic is a color filter array (CFA) for arranging RGB color filters on a square grid of photo sensors. Its particular arrangement of color filters is used in most single-chip digital image sensors used in digital cameras, camcorders, and scanners to create a color image. The filter pattern is $50 \%$ green, $25 \%$ red and $25 \%$ blue, hence is also called RGBG, GRGB, or RGGB.

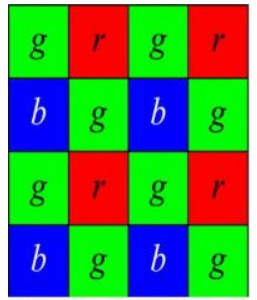

(a)

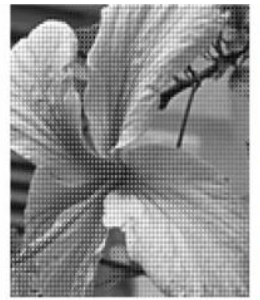

(b)

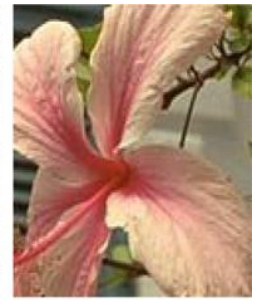

(c)
Fig. 1. Single-sensor imaging concept: (a) Bayer CFA pattern; (b) a CFA image; (c) demosaicked full-color version of (b).

\subsection{Decomposition}

The decomposition is required because there are two problem .First there can be some visible noise residual in the smooth areas. Second there can be some phantom artifacts along edge boundaries with smooth background[1]. Such noise residual and phantom artifacts make the reconstructed color image visually unpleased in some areas. The noise residual in the smooth areas is mainly caused by the relatively low local signal to noise ratio or contrast. Since there are no strong edge structures in the smooth areas, there are fewer significant principal components in the domain, and, hence, the discrimination between noise and signals is not as effective as that in the areas with strong edges. In addition, the training samples for the red, green and blue variables are collected from the local window according to the Bayer pattern. The reason for the phantom artifacts along edge boundaries with smooth background is as follows. The proposed denoising algorithm will use a local training block to estimate the transformation matrix. All the possible samples in the training block are used in the calculation. However, sample structures may change within a block, especially if the block contains object boundaries with smooth background. Involving such samples in the training may lead to much bias in the estimation of transformation matrix and consequently reduce the denoising performance, e.g., generating many phantom artifacts. To overcome the above two problems, we propose two pre processing steps before applying the denoising. First, we decompose the noisy CFA image into two parts: the lowpass smooth image and the high-pass image. Denote by Iv the noisy CFA image. We use a 2-D Gaussian low-pass filter

$$
G\left(x_{i} y\right)=1 \sqrt{2 \pi} \operatorname{sexp}\left(-x^{2}+y^{2} / 2 s^{2}\right)
$$

to smooth Iv.

$$
I_{v}^{l}=I_{v} * G^{*}
$$

The high-pass image is then obtained as

$$
I_{v}^{h}=I_{v}-I_{v}^{l}
$$

The low-pass image will be almost noiseless and most of the noise is contained in the high-pass output, which also contains the image edge structures to be preserved. Since low-pass image is almost noiseless, we do not make further processing on it. The ICA-based CFA denoising scheme will be applied to the high-pass image, where the noise will be dominant in the smooth areas and they can then be better suppressed by LMMSE filtering PCA domain. The final denoised CFA image is obtained as

$$
\hat{\mathrm{I}}=I_{v}^{l}+\widehat{I_{v}^{h}}
$$

\subsection{CFA Image Denoising and Training Sample} Selection

The denoising is done as follows:

1) Set the sizes of variable block and training block.

2) The noise co-variance matrix can then be determined.

3) Perform the training sample selection procedure

Following the underlying layout of the CFA, such as the Bayer pattern considered throughout this a block which consists of at least one red, one green and one blue sample. We call this block the variable block because the elements in this block will be used as the variables in training[2]. For example, Fig. 2 shows the variable block with four elements: one red, one blue and two green samples.

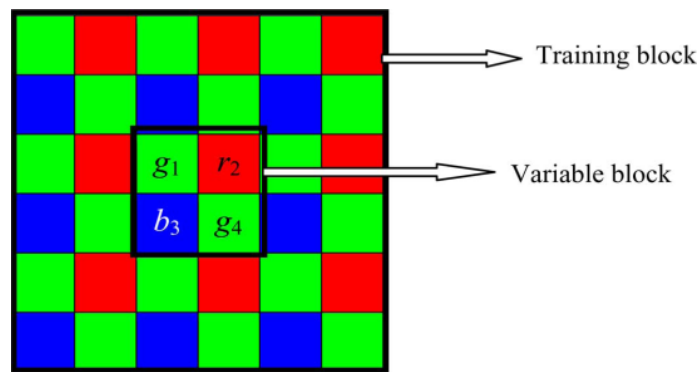

Fig.2 Variable block with four elements 
This training block should be much bigger than the variable block in order to ensure that the statistics of the variables can be reasonably calculated. Wherever any part of the training block can match the variable block, the pixels of that part will be taken as the samples of the variable. The noise residual in the smooth areas is mainly caused by the relatively low local signal to noise ratio or contrast. Since there are no strong edge structures in the smooth areas, there are fewer significant principal components in the PCA domain, and, hence, the discrimination between noise and signals is not as effective as that in the areas with strong edges. In addition, the training samples for the red, green and blue variables are collected from the local window according to the Bayer pattern. The mean values of the variables are computed as the average of the samples and then subtracted from the sample matrix for covariance calculation. However, in this way, the neighboring green and green pixels will not contribute to the mean value estimation of the blue variable, and so do for red and green variables. The biases in mean value estimation will lead to estimation bias of co-variance matrix and, hence, the PCA transformation matrix. The reason for the phantom artifacts along edge boundaries with smooth background is as follows. The proposed denoising algorithm will use a local training block to estimate the transformation matrix. All the possible samples in the training block are used in the calculation. However, sample structures may change within a block, especially if the block contains object boundaries with smooth background. Involving such samples in the training may lead to much bias in the estimation of transformation matrix and consequently reduce the denoising performance, e.g., generating many phantom artifacts.

\subsection{Colour demosaicing}

A demosaicing algorithm is a digital image process used to reconstruct a full color image from the incomplete color samples output from an image sensor overlaid with a color filter array (CFA)[4]-[6].Most modern digital cameras acquire images using a single image sensor overlaid with a CFA, so demosaicing is part of the processing pipeline required to render these images into a viewable format. Many modern digital cameras can save images in a raw format allowing the user to demosaic it using software, rather than using the camera's built-in firmware. In this paper we are using Color demosaicking via directional linear minimum mean square-error estimation [4]. The missing green samples are estimated from the filtered PDS in both horizontal and vertical directions, and the two estimates are optimally fused. The resulting green channel is then used to guide the estimation of the missing red and blue samples. the proposed algorithm is non iterative, fast, and easy to implement

\section{EXPERIMENTAL RESULTS}

We performed simulations in an attempt to confirm the theoretical results above. $512 \times 768$ image is taken to examine the result. We examine the signal-noise ratio $(\mathrm{SNR})$
The SNR is defined as:

SNR 10log (Ps / Pn ) (dB)

where Ps is the power of signals, $\mathrm{Pn}$ is the power of noise.

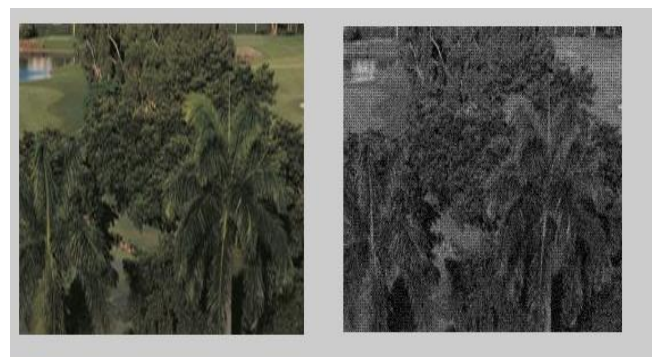

(a)

(b)

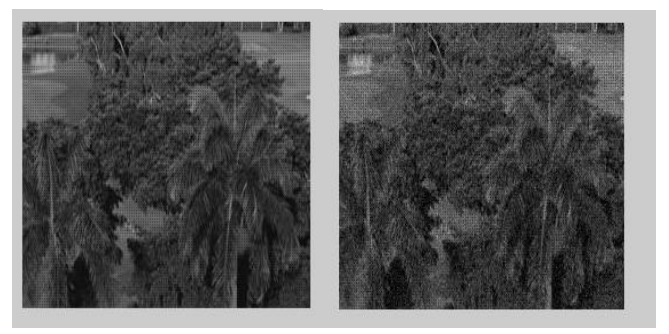

(C)

(d)

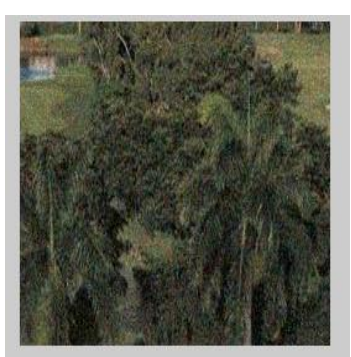

(e)

Fig. 3 Cropped images of the constructed hotelgulf image. (a) Original image; (b) CFA noisy image (c) CFA noiseless image (d) ICA based denoised image (e) is reconstructed by the proposed ICA-based CFA denoising method followed by demosaicking via directional linear minimum mean square-error estimation method .

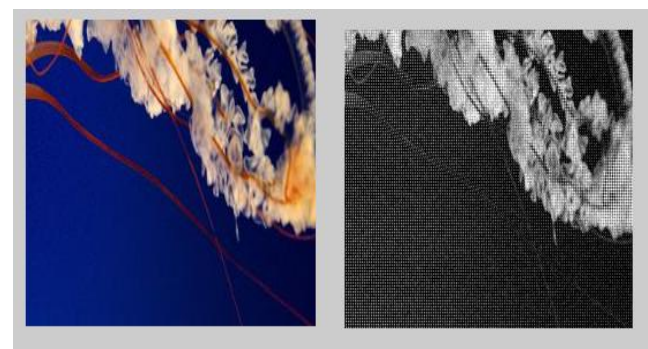

(a)

(b) 


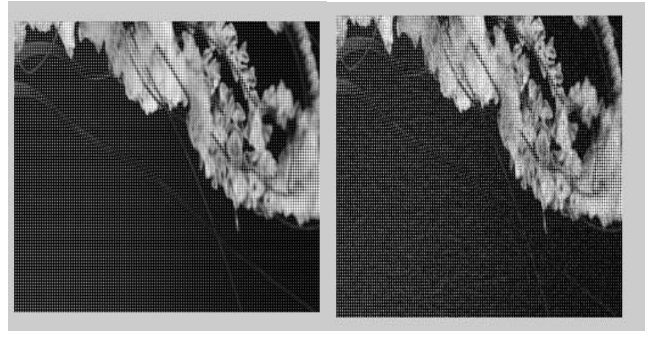

(c)

(d)

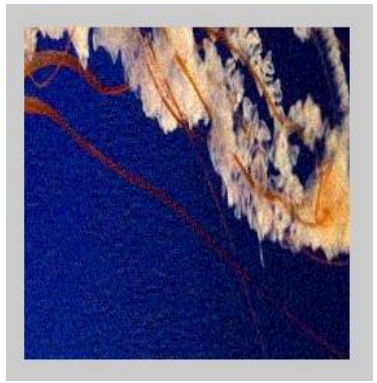

(e)

Fig. 4 Cropped images of the constructed gellyfish image. (a) Original image; (b) CFA noisy image (c) CFA noiseless image (d) ICA based denoised image (e) is reconstructed by the proposed ICA-based CFA denoising method followed by demosaicking via directional linear minimum mean square-error estimation method .

TABLE I

RESULTS OF THE RECONSTRUCTED IMAGES

\begin{tabular}{|c|c|c|c|c|c|}
\hline $\begin{array}{l}\text { image } \\
\mathrm{s}\end{array}$ & $\begin{array}{l}\text { Origin } \\
\text { al } \\
\text { SNR }\end{array}$ & $\begin{array}{l}\text { SNR } \\
\text { after } \\
\text { denois } \\
\text { ing }\end{array}$ & $\mathrm{R}$ & $G$ & B \\
\hline $\begin{array}{l}\text { Hotel } \\
\text { gulf }\end{array}$ & 26.69 & 27.05 & 27.11 & 27.73 & 27.64 \\
\hline $\begin{array}{l}\text { jellyfi } \\
\text { sh }\end{array}$ & 26.67 & 27.70 & 28.13 & 27.94 & 26.82 \\
\hline
\end{tabular}

\section{CONCLUSION}

Performance of denoising algorithms is measured using quantitative performance measures such as peak signal-tonoise ratio (PSNR), signal-to-noise ratio(SNR) as well as in terms of visual quality of the images. Many of the current techniques assume the noise model to be Gaussian. In reality, this assumption may not always hold true due to the varied nature and sources of noise. An ideal denoising procedure requires a priori knowledge of the noise, whereas a practical procedure may not have the required information about the variance of the noise or the noise variance of the noise and the noise model to compare the performance with different algorithms. Noise with different variance values is added in the natural images to test the performance of the algorithm. The performance of different denoising methods can be evaluated by using peak signal-to-noise ratio (PSNR) with respect to the original images. The ICA based CFA denoising method achieves highest PSNR values. While suppressing noise, the proposed scheme preserves very well the fine structures in the image, which are often smoothed by other denoising schemes and the result of colour demosaicing in terms of red, green, blue are analysed .

\section{REFERENCES}

[1] Lei Zhang, Member, IEEE, Rastislav Lukac, Member, IEEE, Xiaolin Wu, Senior Member, IEEE, and David Zhang, Fellow, IEEE "PCA-Based Spatially Adaptive Denoising of CFA Images for Single-Sensor Digital Cameras" IEEE transactions on image processing, vol. 18, no. 4, April 2009.

[2] Tuan-Anh Nguyen, Won-Seon Song, and MinCheol Hong,"Spatially Adaptive Denoising Algorithm for a Single Image Corrupted by Gaussian Noise" IEEE Transactions on Consumer Electronics, Vol. 56, No. 3, August 2010.

[3] L. Zhang, X. Wu, and D. Zhang, "Color reproduction from noisy CFA data of single sensor digital cameras," IEEE Trans. Image Process., vol.16, no. 9, pp. 2184-2197, Sep. 2007

[4] L. Zhang and $\mathrm{X}$. Wu, "Color demosaicking via directional linear minimum mean square-error estimation," IEEE Trans. Image Process., vol.14, no. 12 , pp. 2167-2178, Dec. 2005.

[5] K. Hirakawa and T. W. Parks, "Adaptive homogeneity-directed demosaicing algorithm," IEEE Trans. Image Process., vol. 14, no. 3, pp.360369, Mar. 2005.

[6] K. Hirakawa and T. W. Parks, "Joint demosaicking and denoising,"IEEE Trans. Image Process., vol. 15, no. 8, pp. 2146-2157, Aug.2006.

[7] P. Longère, X. Zhang, P. B. Delahunt, and D. H. Brainard, "Perceptual assessment of demosaicing algorithm performance," Proc. IEEE, vol.90, no. 1, pp. 123-132, Jan. 2002.

[8] R. Kimmel, "Demosaicing: Image reconstruction from CCD samples, 'IEEE Trans. Image Process., vol. 8, no. 9, pp. 1221-1228, Sep. 1999.

[9] Hong-yan Li Guang-long Ren Bao-jin Xiao "Image Denoising Algorithm Based on Independent Component Analysis" World Congress on Software Engineering, 2009.

[10] A.Hyvariene Independent component analysis. John Wiley and Sons, 2001

[11] A.Hyvariene Independent component analysis: algorithm and application. Neural Network, 2000, 13(4) $411 \sim 430$.

[12] Hyvarinen , E.Oja. A fast fixed-point algorithm for independent component analysis . Neural Computation 1997, 9(7)1483 1492.

[13] A.Hyvarinen,A family of fixed-point algorithms for independent component analysis. Proc. Int. Conf.of Acoustics, Speech and Signal Processing 1997 , 3917 3920. 